\title{
Encephalitis as a rare manifestation of novel COVID-19 disease, a para-infection or post-infection complication: A systematic review
}

\author{
Sara Khosraviani', Sepideh Paybast² ${ }^{2}$ Ali Emami ${ }^{1}$ \\ ${ }^{1}$ Research Committee, Qazvin University of Medical Sciences, Qazvin, Iran \\ ${ }^{2}$ Neuroscience Research Center, Qom University of Medical Sciences, Qom, Iran
}

\begin{abstract}
Objective. The novel coronavirus disease (COVID-19) is a global pandemic. Although the main clinical manifestations of the COVID-19 infection are confined to the respiratory system, there is evidence suggesting the COVID-19 potential to develop central nervous system complications. As there are limited reports of encephalitis associated with COVID-19 in the literature, in this systematic review, we will discuss the characteristics of encephalitis in patients with COVID-19.

Material and methods. PubMed, Scopus, ProQuest, ScienceDirect, and Cochrane all from December 1, 2019, to May 25, 2020, were searched for related published articles. The keywords (in the title/abstract) "COVID-19" OR "novel Coronavirus" OR "SARS-CoV-2" AND "encephalitis" OR "meningoencephalitis" OR "encephalopathy" OR neurological complications" were used. We included all the case reports, case series, and letters to the editors.

Outcomes. Through the search strategy, we identified nine related articles about encephalitis associated with COVID-19. Based on our results, although encephalitis is mainly presented in patients with severe COVID-19, it could occur in patients without respiratory symptoms and it might precede the typical features of COVID-19. Additionally, we did not find any exclusive age group or comorbidities predisposed the patients to encephalitis development. Conclusion. An increasing number of reports of COVID-19 patients with encephalitis raises the concern that SARS-CoV-2 is a neuropathogen that could target the brain regardless of respiratory symptoms.
\end{abstract}

Keywords: COVID-19, SARS-CoV-2, encephalitis, meningoencephalitis, encephalopathy

\section{INTRODUCTION}

The novel coronavirus disease (COVID-19) pandemic is caused by SARS-CoV-2, a member of the Coronaviridae family (1). According to the World Health Organization (WHO) website (https:// www.who.int), as of 25th June 2020, COVID-19 has caused 9,561,229 confirmed cases and 485,632 confirmed deaths.

As COVID-19 has more than $80 \%$ genetic similarities to SARS-CoV, it is expected to behave similarly to SARS-CoV binding to the cellular receptor angiotensin-converting enzyme 2 (ACE2) which is expressed in human airway epithelia, lung parenchyma, vascular endothelia, kidney cells, and small intestine cells (1-3). Although the main clinical features of COVID-19 are confined to the respiratory symptoms, the clinical studies have suggested the virus's potential to produce central and peripheral nervous system manifestations (3-5). There is not enough experimental data on the neuropathogenesis of the newly emerging virus. However, the main underlying mechanisms seem to be direct damage to the ACE2 receptors expressed on the blood-brain barrier, the retrograde transmission of the virus through olfactory nerves and bulbs consistent with the clinical observation of anosmia in some patients or other cranial nerves, cytokine cascade-related injury, and hypoxia secondary to cardiorespiratory instability (6-8). 
As the number of patients with COVID-19 increases worldwide, more reports of neurological complications will be evident. Since the onset of the disease, a wide range of neurological complications have been reported as dizziness, headache, encephalopathy and encephalitis, transverse myelitis, epilepsy, hyposmia, dysgeusia, skeletal muscle injury, and Guillain Barre Syndrome (3-8). To the best of our knowledge, encephalitis is reported as a rare complication of COVID-19. The first case of meningoencephalitis associated with COVID-19 was described on 22nd March 2020 in a 24-yearold man presented with acute febrile confusional state and generalized tonic-clonic seizure (9). It is hypothesized that the main pathophysiology is related to the edema secondary to inflammatory injury versus direct injury which itself is considered as a poor prognostic factor (10).

As this rare manifestation portends a worse clinical outcome in patients with COVID-19 and could be even the initial manifestation of the disease, herein we aimed to review the characteristics of encephalitis in association with COVID-19 to provide a more comprehensive knowledge of the management of central nervous system complications of COVID-19.

\section{MATERIAL AND METHODS}

We designed a systematic review to answer three main questions relating to the encephalitis as a serious central nervous system complication of the COVID-19. Our report was written in accordance with the Preferred Reporting Items for Systematic Reviews and Meta-Analyses (PRISMA) guidelines (figure 1).

Our first question focused on the clinical characteristics of encephalitis associated with COVID-19. The second question examined the para-clinical features of encephalitis associated with COVID-19 and the third question investigated the contributing factors in the development of encephalitis in patients with COVID-19.

We did comprehensive research to answer our main concerns. The study was carried out in databases such as PubMed, Scopus, ProQuest, ScienceDirect, and Cochrane all from December 1, 2019, to May 30, 2020, for related published articles. The following MESH keywords (in the title/abstract) were used: "novel coronavirus" OR "COVID-19" OR SARS-CoV-2 AND "encephalitis" OR "encephalopathy" OR "meningoencephalitis" OR "neurological complications".

We assessed the study inclusion criteria based on our three questions. We included all cross-sectional studies, case reports, case series, and letters to editors worldwide to review the characteristic of encephalitis in association with COVID-19 during the COVID-19 pandemic.

After search completion, we used EndNote X9 software to systematically identify and remove duplicate citations. Two reviewers independently reviewed the titles and abstracts and excluded the irrelevant articles. One reviewer focused on the characteristics of encephalitis associated with COVID-19. The second reviewer evaluated the underlying factors contributing to COVID-19 encephalitis development. We subsequently screened the full-text papers to decide whether the articles meet the inclusion criteria. We extracted key data from each study relevant to the specific research questions. The reports concerning the encephalitis as a consequence of other known etiologies, the articles on other central nervous system complications of COVID-19 except for meningoencephalitis, and the peripheral nervous system complications of COVID-19 were excluded. Additionally, the papers written in languages other than English and review papers were excluded. One article was excluded because it was an update on another study.

\section{RESULTS}

204 citations were identified by the researchers. Following the removal of duplicates and title/abstract screening, we retrieved 10 full-text papers of which nine were eligible for inclusion criteria (figure 1). All nine available studies were included for each question.

Table 1 shows a summary of the studies on the reported encephalitis associated with COVID-19 in the literature.

Tables 2 and 3 demonstrates the cerebrospinal fluid (CSF) analysis and neuroimaging findings of the reported patients, respectively. Of note, in the study of Al-olama et al. (11) lumbar puncture was not performed due to the imaging contraindication (evidence of midline shift in brain computed tomography (CT)) and in one study, the SARS-CoV-2 


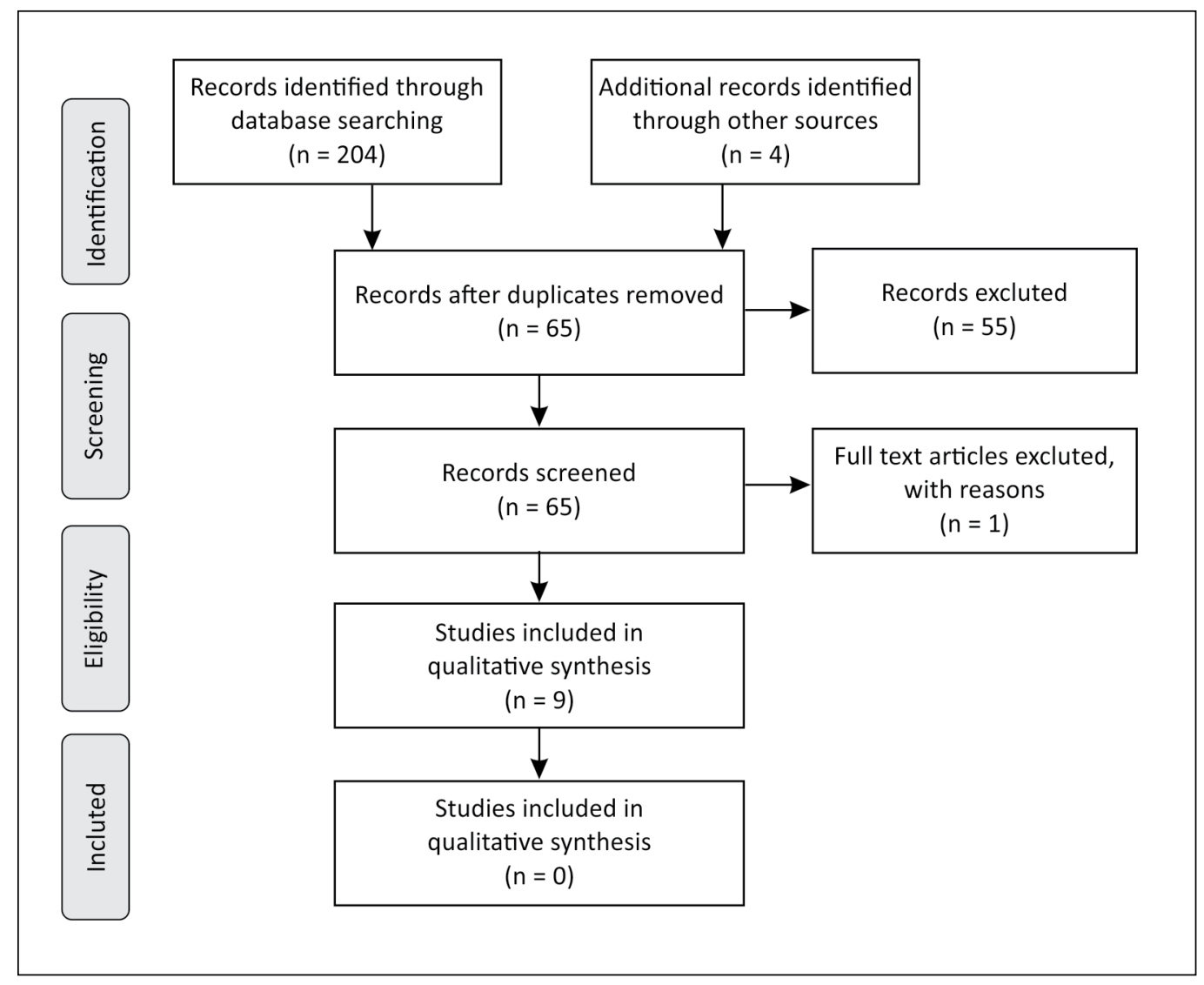

FIGURE 1. Preferred reporting items for systematic reviews and meta-analysis flow chart depicting the selection process of included studies

RNA PCR was not assessed due to low volume sample of CSF (12).

\section{QUESTION ONE}

The main neurological manifestations included fever $(\mathrm{n}=7)$, followed by altered consciousness $(n=5)$, headache $(n=4)$, seizure $(n=4)$, brainstem involvement $(\mathrm{n}=1)$, and unexplained psychosis $(\mathrm{n}=1)$. It should be noted that in one patient the seizure was in a form of status epilepticus (17). In four cases, the neurological manifestations were the initial symptoms $(11,14,16,17)$. In others, the interval between the occurrence of the initial symptoms and encephalitis varied from five to 17 days. These data were not reported for six cases. Additionally, in seven cases, an immune-mediated process was hypothesized $(9,13)$ who had considerable responses to steroid therapy and plasmapheresis.

\section{QUESTION TWO}

CSF polymerase chain reaction of SARS-CoV-2 was performed in all of the cases, except for two
(11,12). Among these, three CSF SARS-Cov-2 PCRs were positive $(9,16,18)$. . Brain imaging including CT and MRI were normal in nine cases. The pathologic involvement in neuroimaging included the right mesial temporal lobe and hippocampus (9), the right frontal lobe (as intracerebral hematoma) (11), the right inferior cerebellar peduncle (12), and bilateral widespread cortical and subcortical white matter involvement (13).

\section{QUESTION THREE}

11 of 13 patients were male. Three patients were in their $60 \mathrm{~s}$, four in their $50 \mathrm{~s}$, and three in their $40 \mathrm{~s}$. 4 patients were $<40$ years old, including one child $(9,1,13,17)$. The disease was severe in nine patients and seven of them needed intubation $(9,13)$ of which one died (13). Hypertension was the most common comorbidity $(\mathrm{n}=5)$, followed by diabetes mellitus $(n=4)$. Leukocytosis was seen in eight cases and one patient had lymphopenia. 


\begin{tabular}{|c|c|c|c|c|c|}
\hline 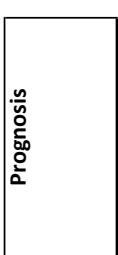 & 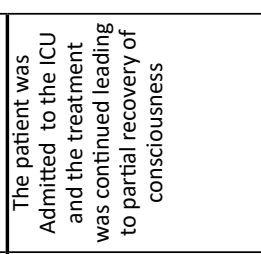 & 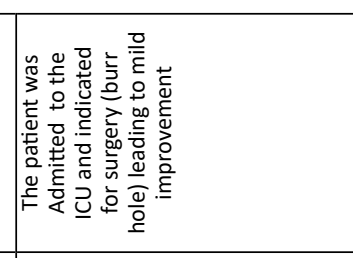 & 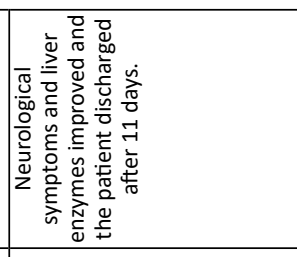 & 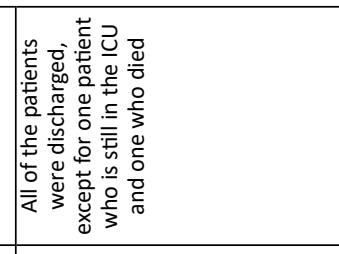 & 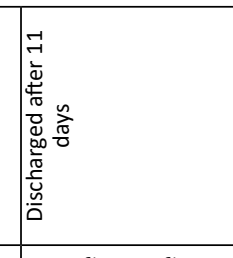 \\
\hline 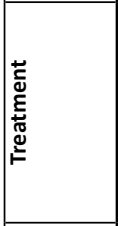 & 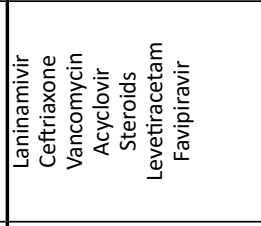 & 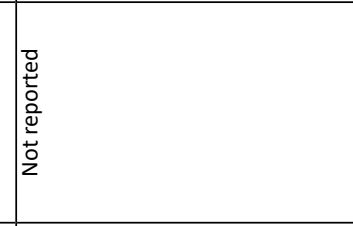 & 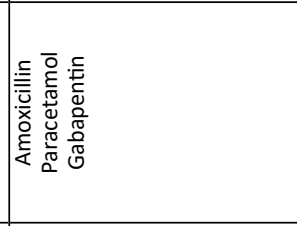 & 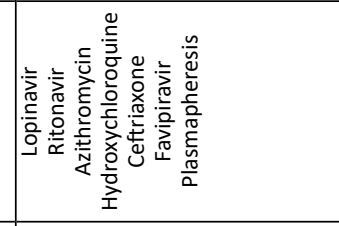 & 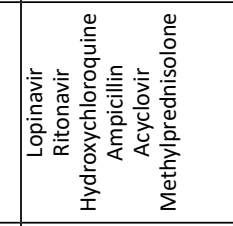 \\
\hline 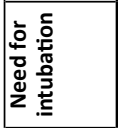 & 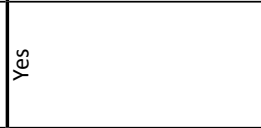 & $\mid \begin{array}{l}\tilde{\partial} \\
\frac{\partial}{2}\end{array}$ & $\mid \begin{array}{l}0 \\
\frac{0}{2} \\
z\end{array}$ & $\stackrel{\ddot{\nu}}{\check{\nu}}$ & 2 \\
\hline 焉 & 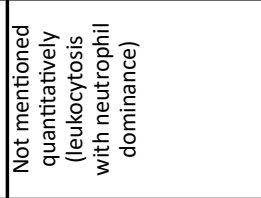 & $\mid \begin{array}{l}\overline{\bar{g}} \\
\bar{\xi} \\
\bar{\partial} \\
z\end{array}$ & 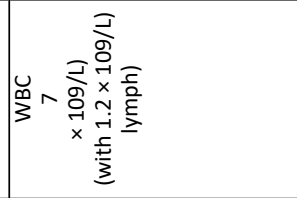 & 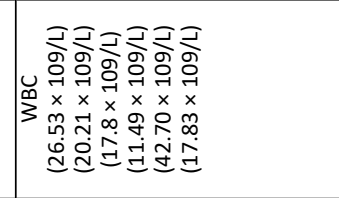 & \begin{tabular}{|l}
$\overline{\bar{\sigma}}$ \\
$\underline{\underline{\xi}}$ \\
$\frac{\partial}{2}$
\end{tabular} \\
\hline 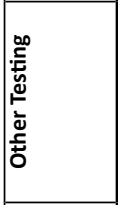 & 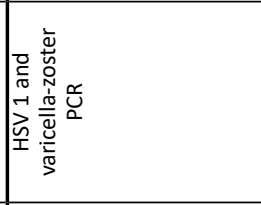 & 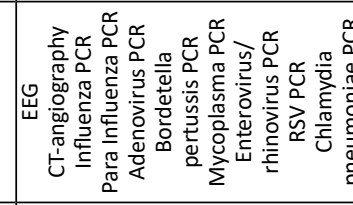 & 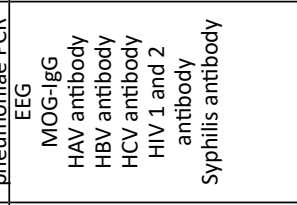 & 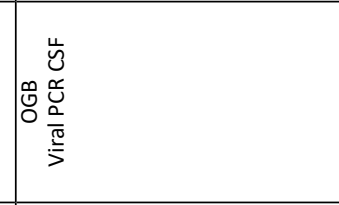 & 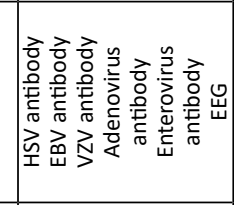 \\
\hline 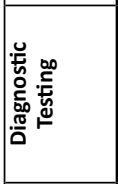 & 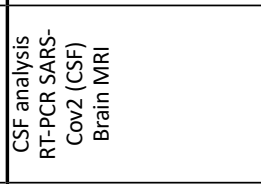 & 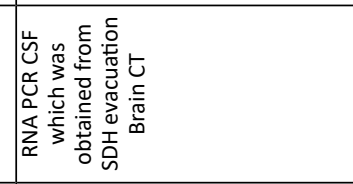 & 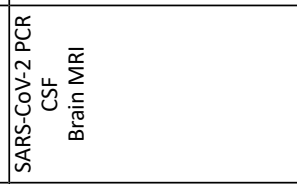 & 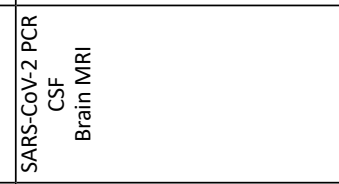 & 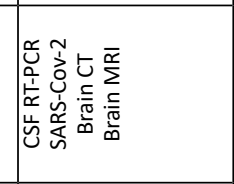 \\
\hline 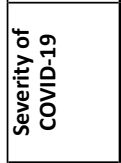 & 这 & 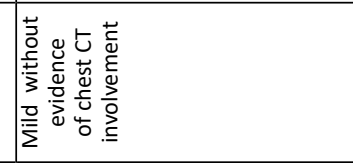 & 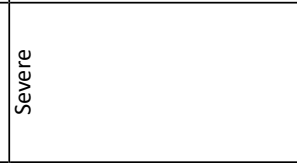 & 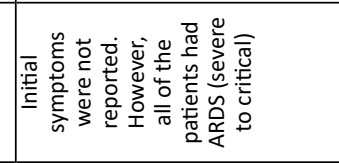 & 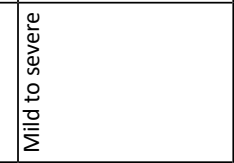 \\
\hline 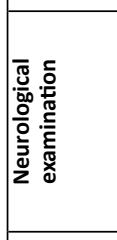 & 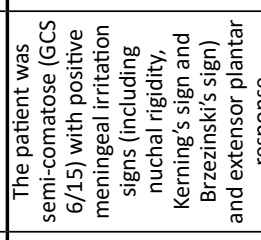 & 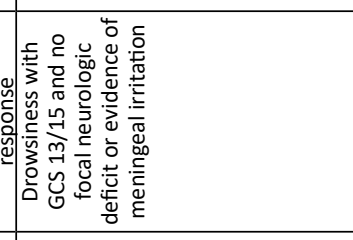 & 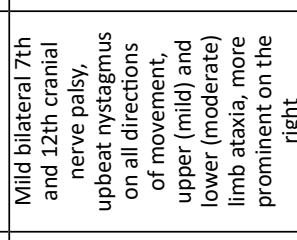 & 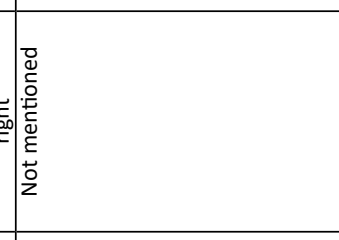 & 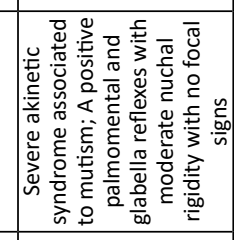 \\
\hline 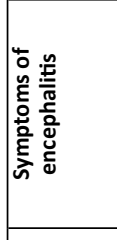 & 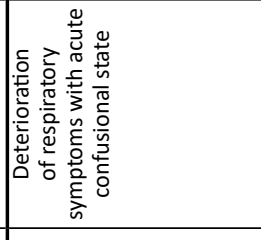 & 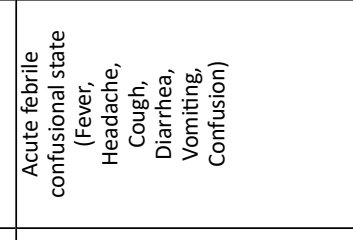 & 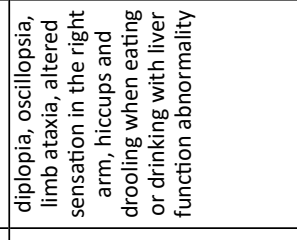 & 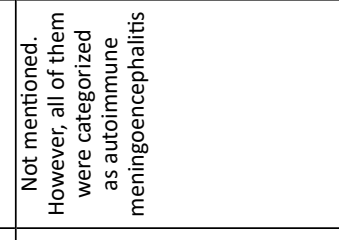 & 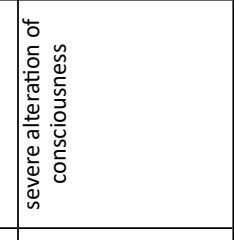 \\
\hline 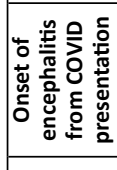 & 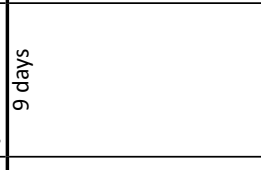 & 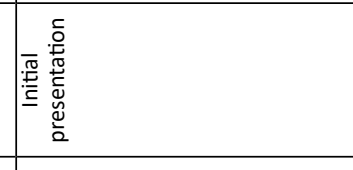 & 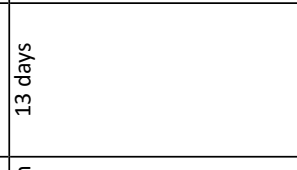 & 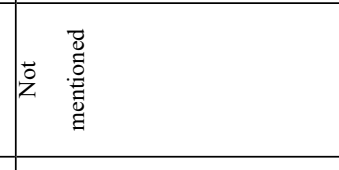 & 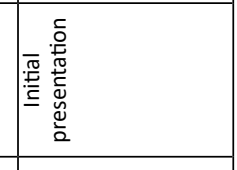 \\
\hline 竞: 苋 & 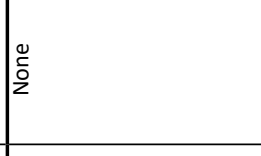 & $\mid \begin{array}{l}\tilde{\Xi} \\
\Sigma\end{array}$ & 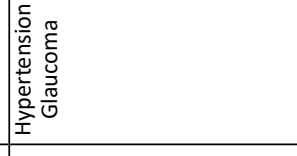 & 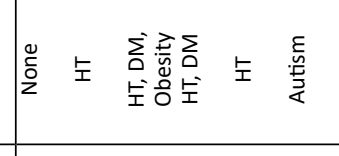 & 夠 \\
\hline 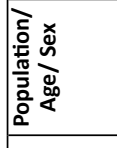 & 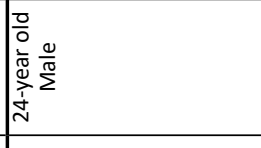 & 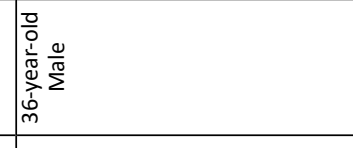 & 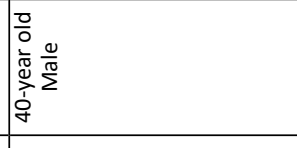 & 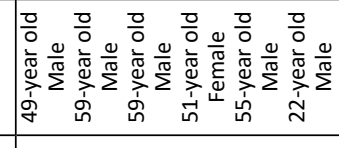 & 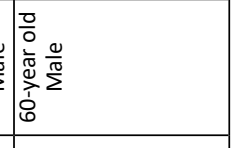 \\
\hline 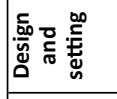 & 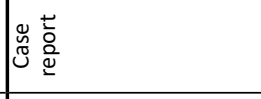 & 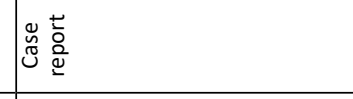 & 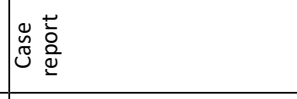 & 总 蒡 & 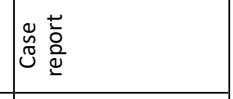 \\
\hline 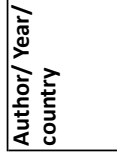 & 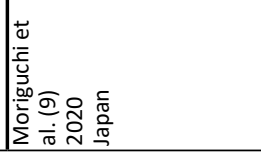 & 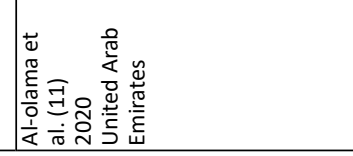 & 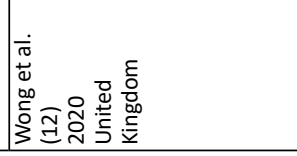 & 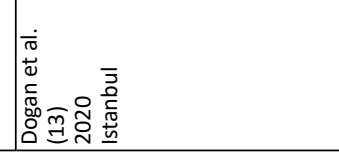 & 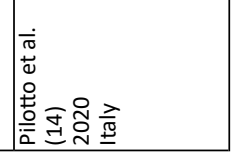 \\
\hline
\end{tabular}




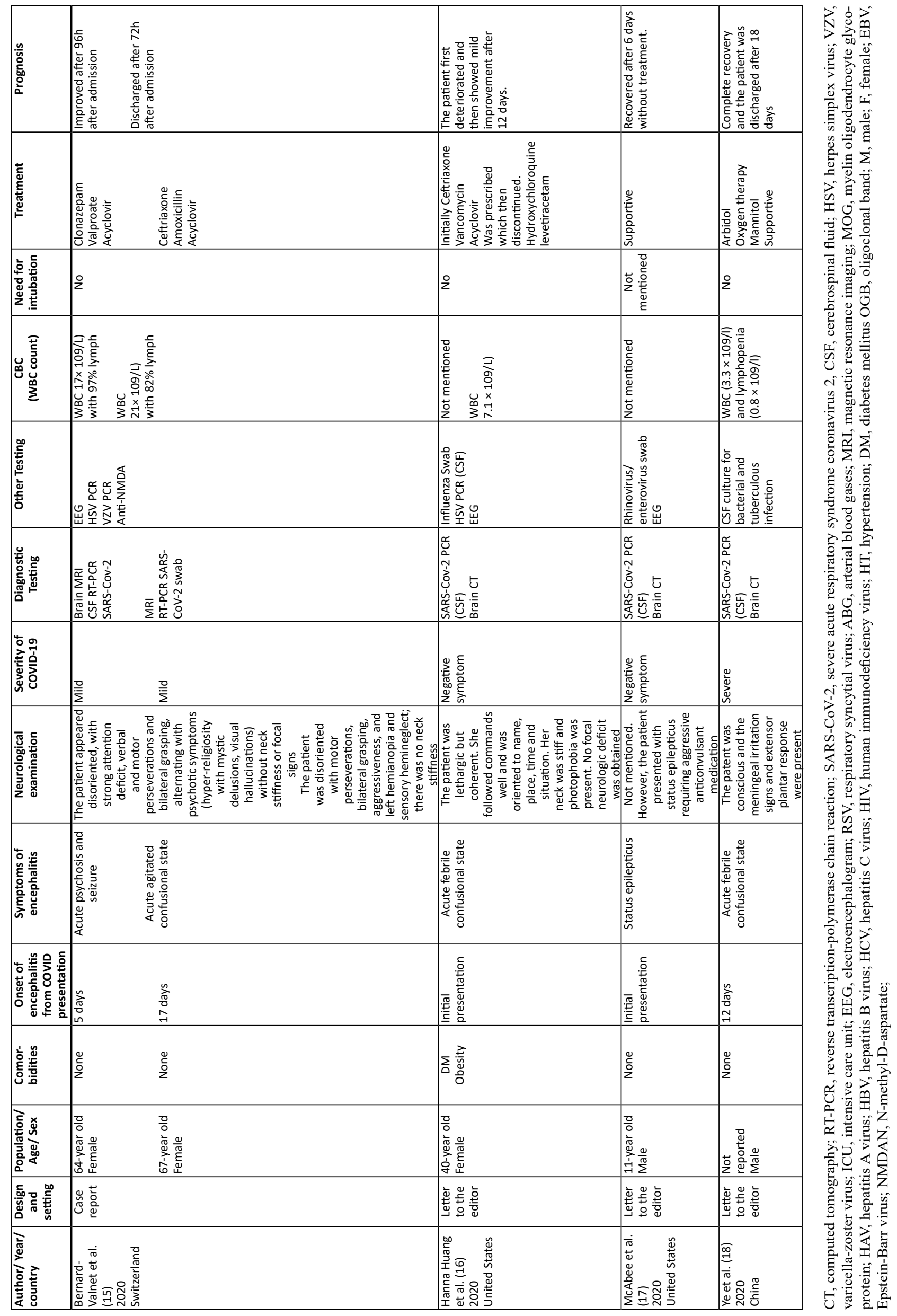


TABLE 2. The results of CSF analysis in included studies considering encephalitis in COVID-19 patients

\begin{tabular}{|c|c|c|c|c|c|c|}
\hline Author & Cell count & Neutrophil & Lymphocyte & Protein (mg/l) & Glucose (mg/l) & SARS-CoV 2 PCR \\
\hline Moriguchi et al. (9) & $12 / \mu \mathrm{l}$ & $10 / \mu \mathrm{l}$ & $2 / \mu l$ & NR & NR & Positive \\
\hline Wong et al. (12) & Normal & Normal & Normal & 423 & NR & $\begin{array}{l}\text { Due to low volume } \\
\text { sample, PCR for } \\
\text { SARS-CoV-2 RNA } \\
\text { was not possible. }\end{array}$ \\
\hline $\begin{array}{l}\text { Dogan et al. (13) } \\
\text { 49-year old M } \\
\text { 59-year old M } \\
\text { 59-year old M } \\
\text { 51-year old F } \\
\text { 55-year old M } \\
\text { 22-year old M }\end{array}$ & $\begin{array}{l}0 \\
0 \\
0 \\
0 \\
0 \\
0\end{array}$ & $\begin{array}{l}0 \\
0 \\
0 \\
0 \\
0 \\
0\end{array}$ & $\begin{array}{l}0 \\
0 \\
0 \\
0 \\
0 \\
0\end{array}$ & $\begin{array}{l}37.6 \\
73.2 \\
65.7 \\
131 \\
52 \\
57 \\
\end{array}$ & $\begin{array}{l}130 \\
201 \\
12 \\
120 \\
67 \\
59\end{array}$ & Negative \\
\hline Pilotto et al. (14) & NR & NR & $18 / \mu \mathrm{L}$ & 69.6 & NR & Negative \\
\hline $\begin{array}{l}\text { Bernard-Valnet et al. (15) } \\
64 \text {-year old F } \\
67 \text {-year old F }\end{array}$ & $\begin{array}{l}17 / \mathrm{mm}^{3} \\
21 / \mathrm{mm}^{3} \\
\end{array}$ & NR & $\begin{array}{l}97 \% \\
89 \% \\
\end{array}$ & $\begin{array}{l}466 \\
461 \\
\end{array}$ & NR & Negative \\
\hline Hanna Huang et al. (16) & NR & NR & NR & NR & NR & Positive \\
\hline McAbee et al. (17) & $16 / \mu \mathrm{l}$ & $8 \%$ & NR & 97 & 92 & Positive \\
\hline Ye et al. (18) & $0.001 \times 109 / 1$ & NR & NR & 270 & 56.52 & Negative \\
\hline
\end{tabular}

$N R$, not reported; $M$, male; $F$, female

TABLE 3. The results of brain CT and brain MRI in included studies considering encephalitis in COVID-19 patients

\begin{tabular}{|c|c|c|}
\hline Author & Brain CT & Brain MRI \\
\hline $\begin{array}{l}\text { Moriguchi et } \\
\text { al. (9) }\end{array}$ & Normal & $\begin{array}{l}\text { DWI showed hyperintensity along the wall of the inferior } \\
\text { horn of the right lateral ventricle. FLAIR images showed } \\
\text { hyper-intense signal changes in the right mesial temporal } \\
\text { lobe and hippocampus with slight hippocampal atrophy. } \\
\text { Contrast-enhanced imaging showed no definite dural } \\
\text { enhancement }\end{array}$ \\
\hline $\begin{array}{l}\text { Al-olama et al. } \\
\text { (11) }\end{array}$ & $\begin{array}{l}\text { Right frontal intracerebral hematoma (with vasogenic } \\
\text { edema and midline shift) associated with subarachnoid } \\
\text { hemorrhage in the ipsilateral Sylvian fissure and } \\
\text { frontal and temporal lobes and a thin, acute subdural } \\
\text { hematoma }\end{array}$ & Not performed \\
\hline Wong et al. (12) & Not mentioned & $\begin{array}{l}\text { An increased signal lesion in the right inferior cerebellar } \\
\text { peduncle, extending to involve a small portion of the } \\
\text { upper cord with evidence of mild edema and associated } \\
\text { micro-hemorrhage in FLAIR sequence }\end{array}$ \\
\hline Dogan et al. (13) & Not mentioned & $\begin{array}{l}\text { Cases 1, 2, and } 6 \text { had pathological findings (cortical or } \\
\text { white matter hyper-intensities, contrast enhancement, } \\
\text { and sulcal hemorrhagic features) } \\
\text { Cases } 3-5 \text { had scans within normal limits (MRI negative) }\end{array}$ \\
\hline Pilotto et al. (14) & Normal & Normal \\
\hline $\begin{array}{l}\text { Bernard-Valnet } \\
\text { et al. (15) }\end{array}$ & Not mentioned & Normal \\
\hline $\begin{array}{l}\text { Hanna Huang et } \\
\text { al. (16) }\end{array}$ & Normal & Not mentioned \\
\hline $\begin{array}{l}\text { McAbee et al. } \\
\text { (17) }\end{array}$ & Normal & Not performed \\
\hline Ye et al. (18) & Normal & Not performed \\
\hline
\end{tabular}

$C T$, computed tomography; MRI, magnetic resonance imaging; DWI, Diffusion-weighted images; FLAIR, fluid-attenuated inversion recovery

\section{DISCUSSION}

In this systematic review of nine studies, we identified that 14 patients with SARS-CoV-2 developed encephalitis which revealed the probable po- tential of the virus to invade the central nervous system. Of note, the early attention to the neurological manifestations of COVID-19 arose from the results of a retrospective case series by Mao et al who revealed a $24 \%$ prevalence of neurological 
complications in 214 cases of COVID-19 (19). Since then, more neurological manifestations have emerged. With regard to the increasing pattern of COVID-19 worldwide, our knowledge of the syndromic complexity of the COVID-19 will be developed.

Despite early discussion over the occurrence of neurological complications in severely ill patients, we demonstrated that three patients with mild respiratory symptoms developed acute confusional state with a final diagnosis of encephalitis associated with COVID-19 (11,15). Interestingly, two patients denied any history of respiratory symptoms and their chest CT was unremarkable $(16,17)$. Moreover, in four patients, the encephalitis preceded the typical features of COVID-19 $(11,14,16,17)$. Taking all considerations into account, our findings not only confirmed the neurotropism of the COVID-19 but also raised the possibility that the brain might be even the only target of SARS-CoV-2.

Since completing the clinical characteristic of the encephalitis, we extracted a wide range of clinical manifestations in addition to the classic symptoms of encephalitis, as the one who developed rhombencephalitis (12) or a case series of six patients with autoimmune encephalitis (13). Moreover, one patient developed unexplained psychosis which was eventually attributed to COVID-19 (15).

On the other hand, we revealed that encephalitis was developed in all age groups ranging from 11 years to 67 years, and the majority of patients did not have serious underlying comorbidity. Hence, it is plausible to expect encephalitis to happen in all patients regardless of the immune status or the age of the patients. Additionally, as in two patients, there was no evidence of respiratory symptoms $(16,17)$, we concluded that the encephalitis might not be exclusively confined to symptomatic cases.

In terms of laboratory investigations, we revealed that the CSF polymerase chain reaction of SARS-CoV-2 was performed in all of the cases, except for two $(11,12)$. Among these, three CSF SARS-CoV-2 PCR was positive $(9,16,18)$. Moreover, the CSF analysis mainly showed increased protein concentration, normal glucose level, and normal or mildly mononuclear pleocytosis. The presence of SARS-CoV-2 in the CSF could be considered as a vantage point to suggest the neuro-invasive potential of SARS-CoV-2 through ACE2-mediated cell entry leading to alterations of the blood-brain barrier (3-5). We also have found traces supporting the role of other neuropathogenesis mechanisms, as in one case, (9) we revealed that despite the negative nasopharyngeal swab PCR, the CSF PCR was positive suggesting the direct pathway of neuro-invasion via olfactory fibers. However, it should be noted that the sensitivity of the available diagnostic tests is not high enough that a negative test rules out the diagnosis of COVID-19.

Additionally, in another study (14), the increased level of inflammatory markers in the CSF including IL-6, IL-8, and TNF- $\alpha$ proposed the role of inflammatory cytokines cascade in SARS-CoV-2 encephalitis development.

In the next step, we investigated the neuroimaging features of the patients with encephalitis. Based on our results, brain imaging was normal in nine cases, which may further support the cytokine related encephalitis hypothesis. Additionally, we did not find a characteristic pattern among patients with abnormal neuroimaging. In one case, the abnormal signal was confined to the right mesial temporal lobe (9). In three cases with autoimmune encephalitis, cortical and white matter hyperintensities and sulcal hemorrhagic features were evident (13). and in the one who developed rhombencephalitis, the brain stem was affected (12). More interestingly, in one patient with an initial complaint of acute febrile confusional state, the brain CT revealed evidence of subdural hematoma with significant vasogenic edema which was not at first attributed to the COVID-19 (11).

Eventually, we assessed the therapeutic strategies and prognosis of patients with encephalitis. Various medications were used. In five cases, no antiviral medications were prescribed. In one case with normal neuroimaging and increased inflammatory markers, a high dose of corticosteroid was prescribed for five days leading to significant clinical improvement (9). This suggests the neuroinflammation induced by SARS-CoV-2 as a mechanism of encephalitis. Similarly, in a case series of six severely ill patients, plasmapheresis was applied leading to clinical improvement in most of the cases (13). This can further support the cytokine storm hypothesis. All of the patients, except one who died, improved.

Overall, although various reports have been published on the central nervous system complica- 
tions of the COVID-19, so far no systematic study has been performed on encephalitis. As the patients with critical COVID-19 might develop an acute confusional state, it is in paramount importance to distinct the superimposed encephalopathic syndrome from the natural course of the disease. Additionally, according to our results, although encephalitis mainly occurred in patients with severe COVID-19, it did not result in worse outcomes as only one patient died throughout hospitalization.

This review highlights the urgent need for research to dedicate more attention to the neurological assessment of patients with COVID-19. Additionally, it is noteworthy to keep in mind COVID-19 as an underlying etiology of unexplained confusional state.

Our study has some limitations. Firstly, encephalitis is such a rare complication associated with COVID-19 with only a few reports in the literature. Secondly, as our knowledge of the neurological presentations associated with COVID-19 is purely descriptive, the imprecise history of the patients, various para-clinical diagnostic tests, and medica-

\section{REFERENCES}

1. Werner C, Scullen T, Mathkour M, Zeoli T, Beighley A, Kilgore MD, et al. Neurological Impact of Coronavirus Disease (COVID-19): Practical Considerations for the Neuroscience Community. World Neurosurgery. 2020.

2. Berger JR. COVID-19 and the nervous system. European Journal of Neurology. 2020;26(2):143-8.

3. Carod-Artal FJ. Neurological complications of coronavirus and COVID-19. Revista de Neurologia. 2020;70(9):311-22.

4. Montalvan V, Lee J, Bueso T, De Toledo J, Rivas K. Neurological manifestations of COVID-19 and other coronavirus infections: A systematic review. Journal of Neurology. 2020;194:105921.

5. Bridwell R, Long B, Gottlieb M. Neurologic complications of COVID-19. Am J Emerg Med. 2020;38(7):1549.e3-1549.e7.

6. Imai Y, Kuba K, Ohto-Nakanishi T, Penninger JM. Angiotensinconverting enzyme 2 (ACE2) in disease pathogenesis. Circulation Journal. 2010;74(3):405-10.

7. Lu R, Zhao X, Li J, Niu P, Yang B, Wu H, et al. Genomic characterisation and epidemiology of 2019 novel coronavirus: implications for virus origins and receptor binding. The Lancet. 2020;395(10224):565-74.

8. Hamming I, Timens W, Bulthuis M, Lely A, Navis G, van Goor H. Tissue distribution of ACE2 protein, the functional receptor for SARS coronavirus. A first step in understanding SARS pathogenesis. The Journal of Pathology: A Journal of the Pathological Society of Great Britain and Ireland. 2004;203(2):631-7.

9. Moriguchi T, Harii N, Goto J, Harada D, Sugawara H, Takamino J, et al. A first case of meningitis/encephalitis associated with SARSCoronavirus-2. International Journal of Infectious Diseases. 2020;94:55-8.

10. Ye M, Ren Y, Lv T. Encephalitis as a clinical manifestation of COVID-19. Brain, Behavior, and Immunity. 2020. tions make the conclusion difficult. More ever, the demographics features of the patients were variously affecting all age groups from childhood to elderly age without significant comorbidity which contribute to a more complex deduction.

\section{CONCLUSIONS}

COVID-19 pandemic has become one of the health crises worldwide. As the disease is spreading worldwide, more neurological complications will be evident. In this systematic review, we identified that encephalitis could develop in all types of COVID-19 without a considerably worse outcome. According to our results, an increasing number of reports of COVID-19 patients with encephalitis raises the concern that SARS-CoV-2 is a neuropathogen that could target the brain regardless of respiratory symptoms.

\section{Authors' contributions}

The complete manuscript was commented on, revised, and approved by all authors.

Conflict of interest: none declared Financial support: none declared

11. Al-Olama M, Rashid A, Garozzo D. COVID-19-associated meningoencephalitis complicated with intracranial hemorrhage: A case report. Acta Neurochirurgica. 2020:1-5.

12. Wong PF, Craik S, Newman P, Makan A, Srinivasan K, Crawford E, et al. Lessons of the month 1: A case of rhombencephalitis as a rare complication of acute COVID-19 infection. Clinical Medicine (London, England). 2020.

13. Dogan L, Kaya D, Sarikaya T, Zengin R, Dincer A, Ozkan Akinci I, et al. Plasmapheresis treatment in COVID-19-related autoimmune meningoencephalitis: Case series. Brain, Behavior, and Immunity. 2020.

14. Pilotto A, Odolini S, Stefano Masciocchi S, Comelli A, Volonghi I, Gazzina S, et al. Steroid-responsive encephalitis in COVID-19 disease. Annals of Neurology. 2020.

15. Bernard-Valnet R, Pizzarotti B, Anichini A, Demars $Y$, Russo E, Schmidhauser $\mathrm{M}$, et al. Two patients with acute meningo-encephalitis concomitant to SARS-CoV-2 infection. Eur J Neurol. 2020.

16. Huang YH, Jiang D, Huang JT. SARS-CoV-2 Detected in Cerebrospinal Fluid by PCR in a Case of COVID-19 Encephalitis. Brain Behav Immun. 2020 Jul;87:149.

17. McAbee GN, Brosgol Y, Pavlakis S, Agha R, Gaffoor M. Encephalitis Associated with COVID-19 Infection in an 11 Year-Old Child. Pediatric Neurology. 2020.

18. Ye M, Ren Y, Lv T. Encephalitis as a clinical manifestation of COVID-19. Brain Behav Immun. 2020.

19. Mao L, Jin H, Wang M, Hu Y, Chen S, He Q, et al. Neurologic manifestations of hospitalized patients with coronavirus disease 2019 in Wuhan, China. JAMA Neurology. 2020. 